\title{
Perioperative Administration of Fibrinogen is Associated with Increased Risk of Postoperative Thromboembolic Complications after Cardiac Surgery

\author{
Carl-Johan Jakobsen ${ }^{1 *}$, Mariann Tang ${ }^{2}$ and Lars Folkersen ${ }^{1}$
}

${ }^{1}$ Department of Anaesthesiology and Intensive Care, Aarhus University Hospital, Skejby, Denmark ${ }^{2}$ Department of Cardiothoracic and Vascular Surgery, AarhusUniversityHospital, Skejby, Denmark

\begin{abstract}
Introduction: Fibrinogen is a key protein in achieving and maintaining haemostasis. Prophylactic fibrinogen has been shown to reduce bleeding after coronary artery bypass graft (CABG) and studies have reported an inverse correlation between preoperative plasma concentration of fibrinogen and the volume of postoperative bleeding after CABG. This indicates that preoperative fibrinogen plasma concentration, even within the normal range, is a limiting factor for haemostasis after cardiac surgery. In theory, fibrinogen might induce a risk for thrombosis and hypercoagulability. The reported adverse effects of fibrinogen administration are few, without clinical significance or blurred by the use of other medications influencing coagulation; the potential adverse effects of prophylactic fibrinogen on larger groups of cardiac surgery patients seem unidentified.
\end{abstract}

Material and methods: All patients undergoing cardiac surgery at Aarhus University Hospital in 2008 and 2009 were obtained from West Denmark Heart Registry $(n=1876)$ and merged with our patient data management system to identify patients receiving fibrinogen, aprotinin and recombinant Vlla perioperatively. The outcomes considered included in-hospital myocardial infarction, stroke and need for dialysis. Model-based Poisson regression analysis was used to estimate adjusted risk ratios to identify independent factors with impact on outcomes.

Results: Independent risk factors $(\mathrm{OR}(95 \% \mathrm{Cl})$ ) for postoperative stroke were preoperative neurological dysfunction: 3.10 (1.46- 6.57), valve replacement: 2.12 (1.09 - 4.12), aortic surgery: 3.57 (1.35 - 9.46) and perioperative fibrinogen infusion: $2.69(1.24-5.87)$. Independent risk factors for postoperative dialysis were s-creatinine > $200 \mu \mathrm{mol} / \mathrm{L}: 8.21$ (4.12 - 16.5), age: 1.16 (1.00 - 1.33), Euroscore defined general-: 1.32 (1.17 1.48), and cardiac state: $1.27(1.12-1.44)$ together with perioperative aprotinin: $3.42(1.74-6.77)$ and fibrinogen infusion: 3.77 (2.02 - 7.03).

Conclusion: This study indicates that perioperative administration of fibrinogen could be associated with increased risk of neurological thromboembolic complication and renal failure and thus have potential thromboembolic side effects. However, further studies are warranted to clarify any causal association between postoperative complications and perioperative infusion of fibrinogen. The results further emphasized that administration of fibrinogen should be in accordance with recommended guidelines and always guided by whole blood coagulation evaluation.

Keywords: AFibrinogen; Thromboembolic complications; Cardiac surgery

\section{Introduction}

Severe bleeding during and after cardiac surgery is a serious and relatively common complication associated with increased morbidity and mortality [1-3]. The risk of postoperative bleeding increases with age and prolonged cardiopulmonary bypass (CPB) [4-5]. Excessive bleeding may be caused by both surgical factors and impaired haemostasis due to enhanced fibrinolysis, platelet dysfunction, haemodilution, acidosis, hypothermia and consumption of coagulation factors in addition to the surgical trauma alone [6-7].

Fibrinogen is a key protein in achieving and maintaining haemostasis [8]. It is converted in plasma by thrombin into a fibrin clot at the site of tissue damage in order to minimize blood loss and initiate tissue repair [9]. Administration of prophylactic fibrinogen has been shown to reduce postoperative bleeding after coronary artery bypass graft (CABG) [10] and previous studies have reported an inverse correlation between preoperative concentration of fibrinogen in plasma and the volume of postoperative bleeding after CABG [1115]. This indicates that preoperative fibrinogen plasma concentration, even within the normal range, is a limiting factor for haemostasis after cardiac surgery. One randomized clinical trial in patients undergoing cystectomy demonstrated reduced bleeding and need of transfusion in the group treated with fibrinogen [16].

In theory, fibrinogen could induce a risk for thrombosis and hypercoagulability. Although the reported adverse effects of fibrinogen administration are few [17], without clinical significance $[10,17]$ or blurred by the use of other medications influencing coagulation such as aprotinin [18-20], the potential adverse effects of fibrinogen on larger groups of cardiac surgery patients seem unidentified.

*Corresponding author: Carl-Johan Jakobsen, Dept. of Anaesthesiology and Intensive Care Aarhus University Hospital, Skejby, 8200 Aarhus N, Denmark, Tel: +45 40105200; Fax: +45 89498809; E-mail: cjj@dadlnet.dk

Received October 03, 2011; Accepted December 08, 2011; Published Decembe 15, 2011

Citation: Jakobsen CJ, Tang M, Folkersen L (2011) Perioperative Administration of Fibrinogen is Associated with Increased Risk of Postoperative Thromboembolic Complications after Cardiac Surgery. J Blood Disord Transfus S1:004. doi:10.4172/2155-9864.S1-004

Copyright: (c) 2011 Jakobsen CJ, et al. This is an open-access article distributed under the terms of the Creative Commons Attribution License, which permits unrestricted use, distribution, and reproduction in any medium, provided the original author and source are credited. 


\section{Materials and Methods}

We conducted this cohort study using population-based healthcare databases. The Danish National Health Service provides free universal tax-financed healthcare to all Danish citizens We used the unique personal civil registration number assigned to all Danish citizens at birth to link individual data across databases [21]. The study was approved by The Danish Data Protection Agency.

\section{Study population}

To identify patients who underwent cardiac surgery at Aarhus University Hospital, Skejby from 1 January 2008 to 31 December 2009 we used computerized data from the Western Denmark Heart Registry (WDHR). WDHR is an internet-based clinical registry covering all adult patients undergoing cardiac surgery in the western part of Denmark [22]. The registry is a valuable research tool providing ongoing longitudinal registration of detailed patient and procedural data and reporting to the registry has been mandatory since January 1999. Detailed patient, surgery, anesthesia, and intensive care-related data are collected prospectively. Data quality is ensured by automatic validation at entry combined with systematic validation procedures and random spot-checks.

We found that 1,937 cardiac surgery procedures were performed during the study period. For patients who underwent more than one procedure during the study period, only the most recent procedure was included. Fifty-nine patients undergoing transcutaneous aortic valve replacement were excluded. Finally, 1,876 patients ( $97 \%$ of the original patient population) were included in the study.

\section{Fibrinogen treatment}

Since January 2003, all perioperative monitoring data and medications have been entered into an electronic patient management system at Aarhus University Hospital, Skejby. We identified all patients receiving perioperative fibrinogen treatment by merging data from the WDHR with data from the patient management system. Administration of other medications with potential thrombogenic effect (aprotinin and recombinant factor VII) was also registered. The indications for fibrinogen use were severe ongoing perioperative bleeding without obvious surgical reason or lack of coagulation factors. During the observation period an algorithm for administration of fibrinogen primarily based on RoTEM ${ }^{\bullet}$ thromboelastometry analysis was introduced and fully implemented during the last 6 months. In all, 168 patients were treated with fibrinogen. Retrospectively, a perioperative bleeding problem was confirmed if patients had been transfused with more than five units of allergenic red blood cells (RBC) or plasma, had had a postoperative bleeding of more than $1000 \mathrm{ml}$ during the first 24 hours in the ICU or were subjected to re-do surgery.

\section{Anaesthesia protocols and perioperative procedures}

According to hospital protocol, all preoperative cardiac medications were continued until the morning of surgery with the exception of angiotensin-converting enzyme inhibitors and platelet inhibitors, including aspirin. However, all patients were evaluated individually and patients with acute coronary syndrome were kept on angiotensinconverting enzyme inhibitors and platelet inhibitors until elective or acute surgery. Patients received standard premedication consisting of benzodiazepines? and paracetamol 60-90 minutes before surgery. In general, anaesthesia was administered intravenously using Propofol, Sufentanil and Rocuronium.
In the operating room, patients were monitored routinely using five-lead ECG, radial and pulmonary artery catheters with or without continuous cardiac output measurement (Swan-Ganz CCO/VIP; Edwards Lifesciences LLC, Irvine, CA), pulse-oximetry, capnography as well as temperature monitoring; Some patients were also monitored by transoesophageal echocardiography.

Routine surgical and cardio-protective techniques, including cold crystalloid cardioplegia with open cardio-pulmonary bypass systems, were used in most patients. The system consisted of tubes with a surface-modifying additive coating, an arterial filter with heparin coating, a hollow fiber membrane oxygenator with a surfacemodifying additive coating, and a venous and cardiotomy reservoir. Patients were maintained normothermic or slightly hypothermic. The majority of patients $(n=1,748)$ received anti-fibrinolytic treatment with tranexamic acid, receiving in total $4 \mathrm{~g}$ or aprotinin $(\mathrm{n}=128)$ during the surgical procedure. At the end of surgery, reperfusion of the heart was performed according to the patient's general condition and time on cross-clamp. There was no standard postoperative treatment regimen for either pharmacological or mechanical support.

\section{Patient and procedure characteristics}

Patients and procedures were characterized primarily by EuroSCORE [23] containing the following variables: age, sex, chronic pulmonary disease, extra cardiac arteriopathy, neurologic dysfunction, previous cardiac surgery, high preoperative serum creatinine $(>200$ $\mu \mathrm{mol} / \mathrm{l})$, active endocarditis, critical preoperative state, unstable angina, recent myocardial infarction (MI), pulmonary hypertension, left ventricular function (three levels), emergency operation, post infarct septal rupture together with type of surgery (4 categories). We computed the EuroSCORE based on these variables [23]. Further preoperative antiplatelet medication and perioperative administration of aprotinin and recombinant factor VIIa (rVIIa)(Novo Seven ${ }^{\circ}$ ) were included in analysis.

\section{Outcome}

The safety outcomes considered included 30-day mortality, postoperative in-hospital MI (5 timers above upper CK-MB level and/ or new Q-wave in ECG), need for postoperative in-hospital dialysis and postoperative in-hospital stroke. Stroke was combined with outcomes of registered transitory ischaemic attack (TIA - lasting less than 24 hours) and registered cerebrovascular attack (CVA - lasting more than 24 hours). Data on 30-day mortality were obtained from the Danish Civil Registration System; the system keeps updated records on vital status, date of death, residence, and migration since 1968 of all Danish citizens.

\section{Statistical analyses}

Univariate analysis was done using student's t-test or $\chi 2$-test. A model-based Poisson regression analysis with robust error variance was used to estimate adjusted (patient, procedure and medicine characteristics) risk ratios to identify independent factors with impact on outcomes. Analyses were performed with MedCalc ${ }^{\oplus}$ software version 11.5.1 (Mariakerke, Belgium). A probability value of $<0.05$ was used to define statistical significance.

\section{Results}

A total of $9.8 \%$ of patients received fibrinogen perioperatively. The retrospective analysis showed that a bleeding problem was present in $89.9 \%$ of fibrinogen patients compared to $19.6 \%$ in the control group 
(Table 1). Further $2.4 \%$ was grouped as receiving fibrinogen due to re-do surgery and $3.0 \%$ due to marginal or low s-fibrinogen. In $4.8 \%$ of patients the indication was unknown.

In the fibrinogen group, $91.1 \%$ of patients received transfusion with blood or blood products compared to $30.3 \%$ in the control group $\left(\mathrm{P}<0.0001, \chi^{2}\right.$-test $)$. The average volume in transfused patients was $2,601 \mathrm{ml} v$ s. $1,238 \mathrm{ml}$ of RBC, $2,273 \mathrm{ml}$ vs. $1,338 \mathrm{ml}$ of plasma and 1.039 $\mathrm{ml}$ vs. $632 \mathrm{ml}$ of platelets (all $\mathrm{P}<0.0001$, T-test).

Selected indicators and outcome parameters are shown in Table 2. The predominant finding was that the patients receiving fibrinogen had a higher EuroSCORE. Patients receiving fibrinogen were more often medicated with platelet inhibitors up to the time of surgery, were more often? treated with perioperative aprotinin and recombinant factor VII (rVIIa) and were more often subjected to aortic surgery and less often to $\mathrm{CABG}$.

With regard to safety outcomes a significantly higher number had a stroke (6.5\% vs. $1.9 \%, \mathrm{P}=0.0003)$ and a higher number needed postoperative dialysis $(13.7 \%$ vs. $3.7 \%, \mathrm{P}<0.0001)$, while no difference was found in postoperative MI and 30-day mortality (Table 2).

The adjusted odds-ratios for postoperative stroke, MI and need for dialysis are shown in Table 3. Independent risk factors (OR (95\% CI)) for postoperative stroke were aortic surgery 3.57 (1.35 - 9.46), preoperative neurologic dysfunction 3.10 (1.46- 6.57), perioperative fibrinogen 2.69 (1.24 - 5.87) and valve surgery $2.12(1.09-4.12)$. The only independent risk factor for developing postoperative MI was critical preoperative cardiac state defined by EuroSCORE parameters, while independent risk factors for need of postoperative dialysis was preoperative s-creatinine $>200 \mu \mathrm{mol} / \mathrm{L} 8.21$ (4.12 - 16.5), perioperative fibrinogen $3.77(2.02-7.03)$ and aprotinin infusion $3.42(1.74-6.77)$ together with EuroSCORE parameters: patient's general preoperative state $1.32(1.17-1.48)$ and cardiac state $1.27(1.12-1.44)$.

When excluding the confounding factors preoperative neurologic dysfunction as a parameter of prior thromboembolic factor and preoperative treatment with platelets inhibitors, the cohort was reduced to 1,540 patients. The adjusted risk ratio for fibrinogen treatment on postoperative stroke, $\mathrm{MI}$ and need for dialysis increased to 3.24 (1.26 $8.36), 2.62(1.05-6.52)$ and $3.95(1.84-8.49)$, respectively.

\section{Discussion}

This study demonstrates an increased risk of postoperative renal insufficiency and higher incidence of stroke associated with administration of fibrinogen concentrate. Fibrinogen is administered to a patient group with higher EuroSCORE undergoing complex surgical procedures, which increase the risk of stroke and dialysis. It should be noted that there is no significant difference in previous neurological dysfunction between the two groups (12.5\% (fibrinogen

\begin{tabular}{|c|c|c|}
\hline Indication/problem & Fibrinogen & Control \\
\hline No patients & 168 & 1708 \\
\hline Peroperative bleeding a) & $7.1 \%$ & $0.9 \%$ \\
\hline Postoperative bleeding a) & $58.9 \%$ & $16.3 \%$ \\
\hline Perioperative bleedinga) & $23.8 \%$ & $2.4 \%$ \\
\hline Re-do surgery ${ }^{b}$ & $36.9 \%$ & $4.7 \%$ \\
\hline Low or marginal low s-fibrinogen ${ }^{c}$ ) & $51.7 \%$ & $25.6 \%$ \\
\hline None $e^{a)}$ & $4.8 \%$ & $79.2 \%$ \\
\hline
\end{tabular}

Table 1: Retrospective analysis of bleeding events distributed on fibrinogen infusion. a) All together patients with bleeding events or not, $\mathrm{P}<0.0001$; b) Fraction of all patients, $\mathrm{P}<0.0001$; $^{\text {c) }}$ Fraction of all measured $\mathrm{s}$-fibrinogen, $\mathrm{P}=0.0152$ (all $X^{2}$-test).

\begin{tabular}{|c|c|c|c|}
\hline Factors & Fibrinogen & Control & p-value ${ }^{c}$ \\
\hline No of patients & 168 & 1.708 & \\
\hline \multicolumn{4}{|l|}{ Preoperative factors } \\
\hline $\begin{array}{l}\text { Preoperative platelets } \\
\text { inhibitors }\end{array}$ & $28(16.7 \%)$ & $159(9.3 \%)$ & 0.0037 \\
\hline Neurologic dysfunction & $21(12.5 \%)$ & $1438.4 \%)$ & 0.0908 \\
\hline S-creatinine $>200 \mu \mathrm{mol} / \mathrm{L}$ & $8(4.8 \%)$ & $54(3.2 \%)$ & 0.3783 \\
\hline Female & $38(22.6 \%)$ & $515(30.2 \%)$ & 0.0506 \\
\hline Age (mean) & $64.4 \pm 14.4$ & $64.6 \pm 13.5$ & 0.8187 \\
\hline Euroscore (mean) & $8.0 \pm 4.3$ & $5.9 \pm 3.7$ & $<0.0001$ \\
\hline $\begin{array}{l}\text { EuroSCORECardiac state } \\
\text { (mean) }\end{array}$ & $1.2 \pm 1.8$ & $1.2 \pm 1.6$ & 0.5517 \\
\hline $\begin{array}{l}\text { Pre op. patient factors comp } \\
\text { (Euroscore) }^{\text {a) }}\end{array}$ & $1.5 \pm 2.1$ & $0.8 \pm 1.4$ & $<0.0001$ \\
\hline $\begin{array}{l}\text { EuroSCORE age/sex } \\
\text { (mean) }\end{array}$ & $2.3 \pm 1.9$ & $2.4 \pm 2.0$ & 0.7298 \\
\hline $\begin{array}{l}\text { EuroSCORE procedure } \\
\text { (mean) }\end{array}$ & $2.6 \pm 2.0$ & $1.4 \pm 1.5$ & $<0.0001$ \\
\hline $\begin{array}{l}\text { Coronary artery bypass } \\
\text { grafting }^{\text {b) }}\end{array}$ & $60(35.7 \%)$ & $999(58.5 \%)$ & $<0.0001$ \\
\hline Aortic valve replacement ${ }^{b}$ ) & $62(36.9 \%)$ & $555(32.5 \%)$ & 0.2824 \\
\hline Mitral valve replacement $\left.{ }^{b}\right)$ & $24(14.3 \%)$ & $167(9.8 \%)$ & 0.0873 \\
\hline Total valve replacement ${ }^{b)}$ & $83(49.4 \%)$ & $712(41.7 \%)$ & 0.0643 \\
\hline Aortic surgery & $35(20.8 \%)$ & $60(3.5 \%)$ & $<0.0001$ \\
\hline $\begin{array}{l}\text { Other than CABG, valve- or } \\
\text { aortic surgery }\end{array}$ & $23(13.7 \%)$ & $243(14.2 \%)$ & 0.7165 \\
\hline $\begin{array}{l}\text { Perioperative re-combinant } \\
\text { fact VII }\end{array}$ & $20(11.9 \%)$ & $3(0.2 \%)$ & $<0.0001$ \\
\hline Peroperative Aprotinin & $19(11.3 \%)$ & $109(6.4 \%)$ & 0.0240 \\
\hline \multicolumn{4}{|l|}{ Postoperative outcomes } \\
\hline $\begin{array}{l}\text { Neurologic dysfunction } \\
\text { (stroke) }\end{array}$ & $11(6.5 \%)$ & $32(1.9 \%)$ & 0.0003 \\
\hline Myocardial infarction & $9(5.4 \%)$ & $48(2.8 \%)$ & 0.1082 \\
\hline Need for dialysis & $23(13.7 \%)$ & $63(3.7 \%)$ & $<0.0001$ \\
\hline 30-day mortality & $9(5.4 \%)$ & $42(2.5 \%)$ & 0.0505 \\
\hline
\end{tabular}

Table 2: Univariate analysis according to administration of fibrinogen. ${ }^{\text {a) }}$ Preop. patient factors comp (EuroSCORE)= total mean individual EuroSCORE for chronic pulmonary disease, extra cardiac arteriopathy, previous cardiac surgery, active endocarditis and critical peroperative state. $\left.{ }^{b}\right)$ Total number is higher than $100 \%$ as combination surgery is not specified. ${ }^{c)}$ Absolute values $X^{2}$-test, means student's t-test.

group) vs. 8.4\% (control). Preoperative reduced renal function defined as creatinine level $>200 \mu \mathrm{mol} / \mathrm{L}$ was also evenly distributed between the groups (4.8\% (fibrinogen group) vs. $3.2 \%$ (control group).

One argument for not using fibrinogen concentrate has been the potential adverse effects related to transfusion of blood products [24]. It is important to notice that fibrinogen concentrate (Haemocomplettan ${ }^{\oplus}$ ) is a plasma-derived product. It is virus inactivated by pasteurization and afterwards purification to reduce risk of an immunological or allergic reaction as well as diminish the risk of transfer of pathogens.

The findings of aprotinin as an independent risk factor for postoperative dialysis is in accordance with previous study [25]. Nineteen $(11 \%)$ of the patients in the fibrinogen group also received aprotinin. Excluding this group did not affect the results, still demonstrating a significantly higher number of patients with stroke or renal failure. Administration of rVIIa has previously been associated with an increase in serious adverse effects; a clinical randomized trial was terminated pre-term due to thromboembolic complications [26]. However, in this study rVIIa alone did neither increase the incidence of thromboembolic complications (neurological dysfunction, MI) nor the risk of dialysis; only a relatively small number of patients were treated 


\begin{tabular}{|l|c|c|c|}
\hline \multicolumn{1}{|c|}{ Factors } & $\begin{array}{c}\text { Neurological } \\
\text { dysfunction }\end{array}$ & $\begin{array}{c}\text { Myocardial } \\
\text { infarction }\end{array}$ & $\begin{array}{c}\text { Postoperative } \\
\text { dialysis }\end{array}$ \\
\hline Age (EuroSCORE) & $1.16(0.97-1.39)$ & $0.97(0.83-1.14)$ & $1.16(1.00-1.33)$ \\
\hline Female & $0.50(0.23-1.11)$ & $1.17(0.63-2.16)$ & $1.02(0.60-1.75)$ \\
\hline Patient factors comp ${ }^{\text {a) }}$ & $0.99(0.83-1.19)$ & $0.90(0.73-1.10)$ & $1.32(1.17-1.48)$ \\
\hline Neurological dysfunction & $3.10(1.46-6.57)$ & $1.68(0.73-3.86)$ & $1.27(0.61-2.62)$ \\
\hline S-creatinine $>200 \mu$ mol/ L & $0.92(0.19-4.38)$ & $0.40(0.05-3.10)$ & $8.21(4.12-16.5)$ \\
\hline Preop. platelets inhibitors & $0.57(0.17-1.97)$ & $0.73(0.30-1.84)$ & $0.55(0.25-1.20)$ \\
\hline Euroscore Cardiac state & $1.13(0.94-1.37)$ & $1.29(1.13-1.49)$ & $1.27(1.12-1.44)$ \\
\hline Valve surgery & $2.12(1.09-4.12)$ & $0.85(0.47-1.52)$ & $1.43(0.84-2.45)$ \\
\hline Aortic surgery & $3.57(1.35-9.46)$ & $2.05(0.72-5.84)$ & $0.98(0.36-2.69)$ \\
\hline $\begin{array}{l}\text { Other than aortic/valve/ } \\
\text { CABG }\end{array}$ & $1.11(0.41-3.01)$ & $0.20(0.05-0.88)$ & $1.52(0.80-2.89)$ \\
\hline Peroperative Aprotinin & $1.10(0.34-3.50)$ & $1.27(0.39-4.12)$ & $3.42(1.74-6.77)$ \\
\hline $\begin{array}{l}\text { Perioperative } \\
\text { NovoSeven }\end{array}$ & $0.74(0.09-6.30)$ & $1.05(0.12-8.89)$ & $0.88(0.20-3.99)$ \\
\hline Perioperative Fibrinogen & $2.69(1.24-5.87)$ & $1.79(0.79-4.05)$ & $3.77(2.02-7.03)$ \\
\hline
\end{tabular}

Table 3: Adjusted odds-ratio and 95\% confidence limits of significant factors from Table 1.a) Patient factors comp (EuroSCORE) = total mean individual EuroS-

COREfor chronic pulmonary disease, extra cardiac arteriopathy, previous cardiac surgery, active endocarditis and critical peroperative state.

with rVIIa. More patients in the fibrinogen group also received rVIIa (11.9\% vs. $0.2 \%)$

European guidelines regarding bleeding in major trauma were recently updated and recommend fibrinogen administration in all cases with plasma level fibrinogen below $1.5 \mathrm{~g} / \mathrm{L}$ or thromboelastometric evidence of fibrinogen deficiency [27]. In this study, 99 of the patients were evaluated with one or more thrombo elastometries. During the study period a standard treatment algorithm for thromboelastometry was established, but some empiric treatment was still ongoing in the study period despite thromboelastometry. All patients had a preoperative coagulation screening with platelet count, activated partial thrombin time (APTT) and INR.

Solomon et al. [28] recently published a retrospective study including 39 elective cardiac surgery patients receiving fibrinogen concentrate with the indication of bleeding. All patients receiving additional coagulation factor concentrates were excluded as well as all patients without measured plasma fibrinogen level prior to fibrinogen infusion and after the number of adverse effects was reported and sufficient increase in plasma fibrinogen was seen after administration of an average $6.5 \mathrm{~g}$; this was larger than the standard dose used in our patient group (2-3 g). One clinical randomized but not blinded trial has been done regarding fibrinogen infusion in cardiac surgery; the study included $20 \mathrm{CABG}$ patients with preoperative fibrinogen $<3.8 \mathrm{~g} / \mathrm{L}[10]$. The primary outcomes were safety related to clinical adverse events and graft occlusion accessed by multi-slice computed tomography (CT). The secondary end points were among others postoperative blood loss and transfusion requirements. Fibrinogen reduced postoperative bleeding but not transfusion needs; one of the patients in the fibrinogen group had an occluded venous graft visualised by CT and another patient had a subclinical pulmonary embolism.

Epidemiological studies have revealed that the risk of arterial and venous thromboembolic events are associated to elevated plasma fibrinogen levels [29-30]. Increased levels of factor VII were also associated with thromboembolism. Previously, recombinant factor VIIa (rVIIa) was used frequently in cardiac surgery but when a clinical randomized trial was carried out it was discontinued due to many serious adverse effects in the group treated with rVIIa [26]. Thus, attention should be made to be careful in the administration of fibrinogen concentrate which was originally developed for treatment of hypofibrinaemia and not for bleeding events in patients with normal levels of fibrinogen. In major bleeding seen in cardiac surgery, fibrinogen is one of the first coagulation factors to deteriorate and haemodilution is known to worsen this coagulopathy [16]. However, fibrinogen concentrate is known to correct a dilutional coagulopathy both in vivo and in vitro studies [16] and a placebo controlled randomized trial by Fenger-Eriksen et al. [16] on 20 patients undergoing cystectomy a reduced bleeding and need of transfusion was demonstrated and no adverse effects were seen in the fibrinogen group.

Alternative methods to increase plasma fibrinogen are administration of either fresh frozen plasma or cryoprecipitate which are both allergenic blood products. The use of cryoprecipitate versus fibrinogen was questioned by Sorensen and Bevan [31] and recommendations favored fibrinogen concentrate especially due to the risk of virus transmission in cryoprecipitate. However, as the haemostatic potency of fresh frozen plasma (FFP) may be insufficient and unpredictable [32] as well as considering the risk of transfusionrelated acute lung injury, the alternatives seem less favorable.

\section{Limitations of the study}

The main strength of our study is its prospective populationbased design with complete follow-up for a broad range of clinical outcomes. Thus, the study has a low risk of selection and information bias. Furthermore, detailed and complete data on patient- and procedure-related characteristics were available. However, this is a historical observational study which implies that conclusions should be interpreted with caution. Although we adjusted for a wide range of possible confounding factors in the analyses, it is evident that patients receiving fibrinogen in the perioperative period to some extent have an adverse prognostic profile compared to patients not treated with fibrinogen. We can therefore not entirely exclude the possibility that residual or unexplained confounding may still influence our results. However, as exclusion of patients with the presumed confounders prior thromboembolic events and preoperative treatment with platelets inhibitors increased the adjusted risk ratio of fibrinogen for all outcomes, the overall hypothesis of fibrinogen as an independent risk factor is further strengthened.

An obvious weakness of this study is that it was not conducted to evaluate the safety of fibrinogen and that data were retrospective. However, the study population is large and data on all patients undergoing surgery were registered in the mandatory database.

Moreover, FFP is an alternative treatment of fibrinogen deficit and it might strengthen the validity of the adjusted odds-ratios to add blood and blood products into the model. However, as a considerable need for transfusion of blood and blood products is a major indication for use of fibrinogen, the use of FFP in the model could increase instead of solve this specific confounder problem. Adding blood and blood products to the odds-ratio model gives a slightly reduced OR for fibrinogen: 1.75 for neurological dysfunction and 2.43 for need of postoperative dialysis; only the latter remains statistically significant.

\section{Conclusion}

This study suggests that administration of fibrinogen concentrate could increase the risk of neurological thromboembolic complication 
Citation: Jakobsen CJ, Tang M, Folkersen L (2011) Perioperative Administration of Fibrinogen is Associated with Increased Risk of Postoperative Thromboembolic Complications after Cardiac Surgery. J Blood Disord Transfus S1:004. doi:10.4172/2155-9864.S1-004

Page 5 of 5

and renal failure and thus has a potential thromboembolic side effect. However, further studies are warranted to clarify any causal association between postoperative complications and perioperative infusion of fibrinogen. The results further emphasizes that administration should be in accordance with recommended guidelines and always be guided by whole blood coagulation evaluation.

\section{References}

1. Moulton MJ, Creswell LL, Mackey ME, Cox JL, Rosenbloom M (1996) Reexploration for bleeding is a risk factor for adverse outcomes after cardiac operations. J Thorac Cardiovasc Surg 111: 1037-1046.

2. Spiess BD (2004) Transfusion of blood products affects outcome in cardiac surgery. Semin Cardiothorac Vasc Anesth 8: 267-281.

3. Dacey LJ, Munoz JJ, Baribeau YR, Johnson ER, Lahey SJ, et al. (1998) Reexploration for hemorrhage following coronary artery bypass grafting incidence and risk factors. Northern New England Cardiovascular Disease Study group. Arch Surg 133: 442-447.

4. Despotis GJ, Filos KS, Zoys TN, Hogue CW Jr, Spitznagel E, et al. (1996) Factors associated with excessive postoperative blood loss and hemostatic transfusion requirements: a multivariate analysis in cardiac surgery patients. Anesth Analg 82: 13-21.

5. Kestin AS, Valeri CR, Khuri SF, Loscalzo J, Ellis PA, et al. (1993) The platele function defect of cardiopulmonary bypass. Blood 82: 107-117.

6. Despotis GJ, Avidan MS, Hogue CW Jr (2001) Mechanisms and attenuation of hemostatic activation during extracorporeal circulation. Ann Thorac Surg 72 : S1821-S1831.

7. Paparella D, Brister SJ, Buchanan MR (2004) Coagulation disorders of cardiopulmonary bypass: a review. Intensive Care Med 30: 1873-1881.

8. Mosesson MW (2005) Fibrinogen and fibrin structure and functions. J Thromb Haemost 3: 1894-904.

9. Scheraga HA (2004) The thrombin-fibrinogen interaction. Biophys Chem 112 117-130.

10. Karlsson M, Ternström L, Hyllner M, Baghaei F, Flinck A, et al. (2009) Prophylactic fibrinogen infusion reduces bleeding after coronary artery bypass surgery. A prospective randomised pilot study. Thromb Haemost 102: 137-144.

11. Aljassim O, Karlsson M, Wiklund L, Jeppsson A, Olsson P, et al. (2006) Inflammatory response and platelet activation after off-pump coronary artery bypass surgery. Scand Cardiovasc J 40: 43-48.

12. Blome M, Isgro F, Kiessling AH, Skuras J, Haubelt H, et al. (2005) Relationship between factor XIII activity, fibrinogen, haemostasis screening tests and postoperative bleeding in cardiopulmonary bypass surgery. Thromb Haemost 9: 1101-1107.

13. Karlsson M, Ternstrom L, Hyllner M, Baghaei F, Nilsson S, et al. (2008) Plasma fibrinogen level, bleeding, and transfusion after on-pump coronary artery bypass grafting surgery: a prospective observational study. Transfusion 48 : 2152-2158.

14. Liu G, McNicol PL, McCall PR, Bellomo R, Connellan J, et al. (2000) Prediction of the mediastinal drainage after coronary artery bypass surgery. Anaesth Intensive Care 28: 420-426.

15. Wahba A, Rothe G, Lodes H, Barlage S, Schmitz G, et al. (1997) Predictors of blood loss after coronary artery bypass grafting. J Cardiothorac Vasc Anesth 11: 824-827.

16. Fenger-Eriksen C, Jensen TM, Kristensen BS, Jensen KM, Tonnesen E, et al (2009) Fibrinogen substitution improves whole blood clot firmness after dilution with hydroxyethyl starch in bleeding patients undergoing radical cystectomy: a randomized, placebo-controlled clinical trial. J Thromb Haemost 7: 795-802.

17. Dickneite G, Pragst I, Joch C, Bergman GE (2009) Animal model and clinica evidence indicating low thrombogenic potential of fibrinogen concentrate (Haemocomplettan P). Blood Coag Fibrinolysis 20: 535-540.

This article was originally published in a special issue, Hematologic Oncology: Diagnosis \& Therapeutics handled by Editor(s). Dr. Ulrich Mahlknecht, Saarland University, Germany
18. Weinkove R, Rangarajan S (2008) Fibrinogen concentrate for acquired hypofibrinogenaemic states. Transfus Med 18: 151-157.

19. Langer F, Steinmetz O, Marx G, Amirkhosravi A, Eifrig B, et al. (2007) Aprotininassociated hemolytic thrombotic microangiopathy in a patient with acute myelogenous leukemia (AML) and systemic coagulopathy. Am J Hematol 82 : 1122-1124.

20. Mangano DT, Tudor IC, Dietzel C (2006) The risk associated with aprotinin in cardiac surgery. N Engl J Med 354: 353-365.

21. Pedersen CB, Gøtzsche H, Møller JO, Mortensen PB (2006) The Danish Civil Registration System. A cohort of eight million persons. Dan Med Bull 53: 441 449

22. Schmidt M, Maeng M, Jakobsen CJ, Madsen M, Thuesen L, et al. (2010) Existing data sources for clinical epidemiology: The Western Denmark Heart Registry. Clin Epidemiol 2: 137-144.

23. Roques F, Nashef SAM, Michel P, Gauducheau E, de Vincentiis C, et al. (1999) Risk factors and outcome in European cardiac surgery: analysis of the EuroSCORE multinational database of 19030 patients. Eur J Cardiothorac Surg 15: 816-823.

24. Rahe-Meyer N, Sørensen B (2011) Fibrinogen concentrate for management of bleeding. J Thromb Haemost 9: 1-5.

25. Jakobsen CJ, Søndergaard F, Hjortdal V, Johnsen SP (2009) Use of aprotinin in cardiac surgery: effectiveness and safety in a population-based study. Eur Cardiothorac Surg 36: 863-868.

26. Gill R, Herbertson M, Vuylsteke A, Olsen PS, von Heymann C, et al. (2009) Safety and efficacy of recombinant activated factor VII: a randomized placebocontrolled trial in the setting of bleeding after cardiac surgery. Circulation 120 21-27.

27. Rossaint R, Bouillon B, Cerny V, Coats TJ, Duranteau J, et al. (2010) Management of bleeding following major trauma: An updated European Guideline. Crit Care 14: R52.

28. Solomon C, Pichlmaier U, Schoechl H, Hagl C, Raymondos K, et al. (2010) Recovery of fibrinogen after administration of fibrinogen concentrate to patients with severe bleeding after cardiopulmonary bypass surgery. Br J Anaesth 104 555-562.

29. Danesh J, Lewington S, Thompson SG, Lowe GDO, Collins R, et al. ( 2005) Plasma fibrinogen level and the risk of major cardiovascular diseases and nonvascular mortality: an individual participant meta-analysis. JAMA 294 1799-809.

30. Flinterman LE, van HylckamaVlieg A, Rosendaal FR, Doggen CJ (2010) Venous thrombosis of the upper extremity: effect of blood group and coagulation factor levels on risk. Br J Haematol 149: 118-123.

31. Sørensen B, Bevan D (2010) A critical evaluation of cryoprecipitate for replacement of fibrinogen. $\mathrm{Br} \mathrm{J}$ Haematol 149: 834-843.

32. Stanworth SJ, Brunskill SJ, Hyde CJ, McClelland DB, Murphy MF (2004) Is fresh frozen plasma clinically effective? A systematic review of randomized controlled trials. Br J Haematol 126: 139-152. 